\title{
Suspensão ao músculo frontal com politetrafluoretileno para o tratamento da blefaroptose
}

\author{
Frontalis suspension with polytetrafluorethylene for the treatment of \\ blepharoptosis
}

Juliana Silvério ${ }^{1}$

Débora Mayumi Sugano ${ }^{2}$

Lúcia Miriam Dumont Lucci ${ }^{3}$

José Ricardo Carvalho Lima Rehder ${ }^{4}$

\section{RESUMO}

Objetivo: Relatar a experiência com o uso do fio de politetrafluoretileno nas cirurgias de suspensão ao músculo frontal para correção de blefaroptose. Métodos: Foram estudados todos os casos de blefaroptose grave submetidos à cirurgia pela técnica de suspensão ao músculo frontal com o fio de politetrafluoretileno, no período de fevereiro de 2003 a abril de 2007. Foram realizadas 36 cirurgias em 23 pacientes, a média de seguimento foi de 15,8 meses (variando de 3 a 36 meses). A técnica cirúrgica utilizada foi a descrita por Fox. Resultados: Entre as causas de blefaroptose foram encontradas: congênita em 20 (86,95\%) pacientes, blefarofimose em $2(8,69 \%)$ pacientes e traumática em $1(4,35 \%)$ paciente. Na primeira semana de pós-operatório, $6(26,08 \%)$ pacientes referiram assimetria palpebral, $4(17,39 \%)$ notaram edema local, $3(13,04 \%)$ pacientes apresentaram granuloma no local do fio e $1(4,35 \%)$ paciente apresentou celulite facial na região frontal unilateral. Após 3 meses de seguimento, $3(13,04 \%)$ pacientes referiram assimetria palpebral, e em $1(4,35 \%)$ paciente persistia o granuloma. Conclusão: $O$ politetrafluoretileno Modelo CV3, 6.0 (Gore-Tex ${ }^{\circledR}$; W.L. Gore \& Associates Inc, Flagstaff, AZ, EUA) é um material adequado com bons resultados funcionais $(86,9 \%)$, baixos índices de complicação $(4,35 \%)$ e insatisfação $(13,4 \%)$, podendo ser uma alternativa em relação à fáscia lata, na cirurgia de suspensão ao frontal para tratamento de ptose palpebral grave.

Descritores: Blefaroplastia/métodos; Blefaroptose/cirurgia; Politetrafluoretileno/utilização; Politetrafluoretileno/efeitos adversos

\section{INTRODUCÃO}

Blefaroptose é a condição em que, na posição primária do olhar, a margem da pálpebra superior encontra-se posicionada em um nível mais baixo que o normal ${ }^{(1-2)}$. A posição normal é $2 \mathrm{~mm}$ abaixo do limbo superior $^{(3)}$. Anormalidades funcionais que podem afetar a visão em pacientes com blefaroptose congênita incluem estrabismo, privação de estímulos, erros refracionais altos, astigmatismo, anisometropia e anormalidades de superfície, onde todas podem levar a ambliopia e perda de binocularidade nos pacientes pediátricos ${ }^{(4-9)}$. A sequência mais aceita no tratamento da ambliopia seria a correção de estrabismo e posteriormente a correção da ptose, no entanto em casos de ptose grave onde há ambliopia por privação de estímulos a correção da ptose deve ser feita antes ${ }^{(4-9)}$.

A técnica da suspensão ao músculo frontal é o tratamento de eleição nas ptoses graves (>3 mm) com ausência ou má função do músculo levan- 
tador da pálpebra superior ( $<4 \mathrm{~mm})$, e é a que mais se aplica nos casos de ptose miogênica congênita e adquirida (miastenia, miopatia mitocondrial), neurogênica (paralisia do III par craniano, síndrome de Horner) e pós-traumática ${ }^{(10)}$. Essa técnica foi inicialmente descrita por Payr em $1909^{(11-13)}$, reintroduzida por Wright em $1922^{(11,14)}$ e modificada por Crawford em $1956^{(12)}$ e Fox em $1963^{(15)}$.

São usadas três classes de material: autólogo, heterólogo e aloplástico. A fáscia lata autóloga foi considerada o material de eleição ${ }^{(12-13)}$, porém sua obtenção acarreta aumento no tempo cirúrgico, formação de cicatriz no segundo sítio cirúrgico e dificuldade de deambulação no pós-operatório recente. Com o tempo também se passou a utilizar fáscia lata heteróloga liofilizada ${ }^{(16)}$ e materiais sintéticos como o poliéster, nylon, supramida ou silicone ${ }^{(17-19)}$. As complicações que podem ocorrer são recorrência da ptose, infecção, formação de granulomas ou extrusão do material.

A partir de $1987^{(20)}$ tem-se usado o politetrafluoretileno expandido (PTFEe) para a correção da blefaroptose. Trata-se de um material poroso, inextensível, inerte e biocompatível, que tem sido empregado em cirurgia vascular e abdominal, com bons resultados ${ }^{(19,21-22)}$.

Sant'Anna et al., em 1994, relataram o uso do PTFEe para o tratamento da blefaroptose grave ${ }^{(11)}$.

O presente estudo relata a experiência com o uso do fio de politetrafluoretileno nas cirurgias de suspensão ao músculo frontal para correção de blefaroptose, realizadas pelo Setor de Plástica Ocular da Disciplina de Oftalmologia da Faculdade de Medicina do ABC, durante o período de janeiro de 2003 a abril de 2007.

\section{MÉTODOS}

Foram estudados todos os casos de blefaroptose grave submetidos à cirurgia pela técnica de suspensão ao músculo frontal com o fio de politetrafluoretileno, realizadas pelo Setor de Plástica Ocular. No período de fevereiro de 2003 a abril de 2007, foram realizadas 36 cirurgias em 23 pacientes, com seguimento mínimo de três meses no pós-operatório.

A técnica cirúrgica utilizada foi a descrita por Fox $^{(15)}$. Sob anestesia geral, são realizadas cinco incisões de $3 \mathrm{~mm}$ de comprimento sendo: 2 incisões acima da linha dos cílios da pálpebra superior e tendo o limbo como referência; 3 incisões na região frontal, onde 2 incisões estão próximas à margem do supercílio e um pouco mais afastadas que as palpebrais, e uma incisão central cerca de 15 a $20 \mathrm{~mm}$ acima do supercílio. As cinco incisões formam a figura de um pentágono.

As incisões são feitas com bisturi lâmina $\mathrm{n}^{\circ} 15$ e aprofundadas até o tarso ( 2 inferiores) e periósteo ( 3 superiores). O fio de Gore-Tex ${ }^{\oplus}$ Modelo CV3, 6.0 (W.L. Gore \& Associates Inc, Flagstaff, AZ, EUA) é passado no plano submuscular utilizando-se a agulha de Wright. A figura 1 mostra o fio passado na região pré-tarsal. As pontas do fio são exteriorizadas pela incisão superior e tracionadas até que a pálpebra fique na posição desejada levemente hipercorrigida (Figura 2). É realizado o anodamento do fio e as incisões de pele são suturadas com pontos simples de fio seda 6-0.

Ao receber alta hospitalar o paciente foi orientado a realizar repouso, compressas frias sobre a região operada, colírio lubrificante no olho operado quatro vezes ao dia e retornar ao ambulatório com 1, 4 e 12 semanas de pós-operatório.

\section{RESULTADOS}

No período de fevereiro de 2003 a abril de 2007, foram realizadas cirurgias de suspensão ao músculo frontal em 23 pacientes, sendo $18(78,26 \%)$ do sexo masculino e 15 $(21,74 \%)$ do sexo feminino. A idade dos pacientes variou de 4 a 55 anos (média 16,43) e o seguimento do pós-operatório variou de 3 a 36 meses (média 15,87). Foram realizadas 36 cirurgias, sendo bilateral em $13(56,52 \%)$ pacientes e unilateral em $10(43,48 \%)$ pacientes, sendo somente no olho direito em $4(17,39 \%)$ pacientes, e no olho esquerdo em $6(26,08 \%)$ pacientes. A figura 3 mostra o resultado da técnica da suspensão frontal com fio de PTFEe (Gore-Tex ${ }^{\circledR}$ Modelo CV3, 6.0 W.L. Gore \& Associates Inc, Flagstaff, AZ, EUA) num paciente com ptose congênita bilateral grave.

Entre as causas da blefaroptose foram encontradas: congênita em $20(86,95 \%)$ pacientes, blefarofimose em $2(8,69 \%)$ pacientes e traumática em 1 (4,35\%) paciente.

As complicações na primeira semana de pós-operatório foram: assimetria palpebral em 6 pacientes (26,08\%), edema local em 4 pacientes $(17,39 \%)$, granuloma no local do fio em 3 pacientes $(13,04 \%)$ e celulite facial na região frontal em 1 paciente $(4,35 \%)$.

Os pacientes que apresentaram granulomas foram orientados a realizar compressas mornas, massagem e aplicação de pomada dermatológica de antibiótico e corticosteróide quatro vezes ao dia, sobre o local da lesão.

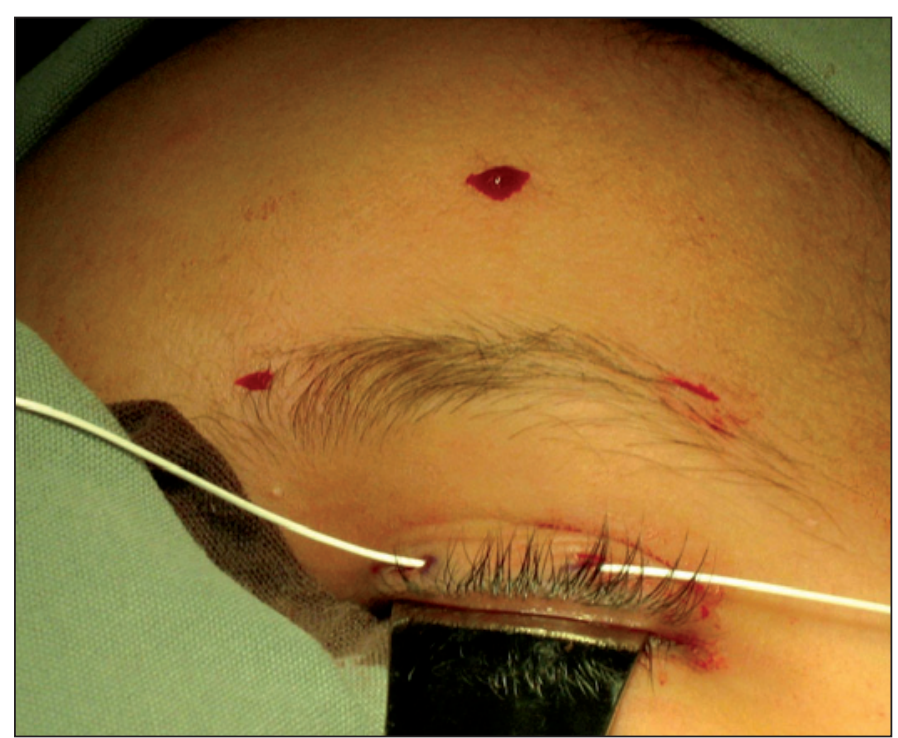

Figura 1 - Fio de Gore-Tex ${ }^{\circledR}$ passado no plano pré-tarsal 
Após três meses de seguimento, $3(13,04 \%)$ pacientes referiram assimetria palpebral e em $1(4,35 \%)$ paciente persistia o granuloma. Os demais referiram satisfação estética e visual.

\section{DISCUSSÃO}

A correta avaliação pré-operatória da ptose é de extrema importância na escolha da técnica cirúrgica. A suspensão ao músculo frontal está indicada nos casos onde a função do músculo levantador da pálpebra superior é menor do que $4 \mathrm{~mm}$.

Atualmente a técnica de Fox $^{(15)}$ é a mais utilizada por permitir uma melhor e mais simétrica posição palpebral final.

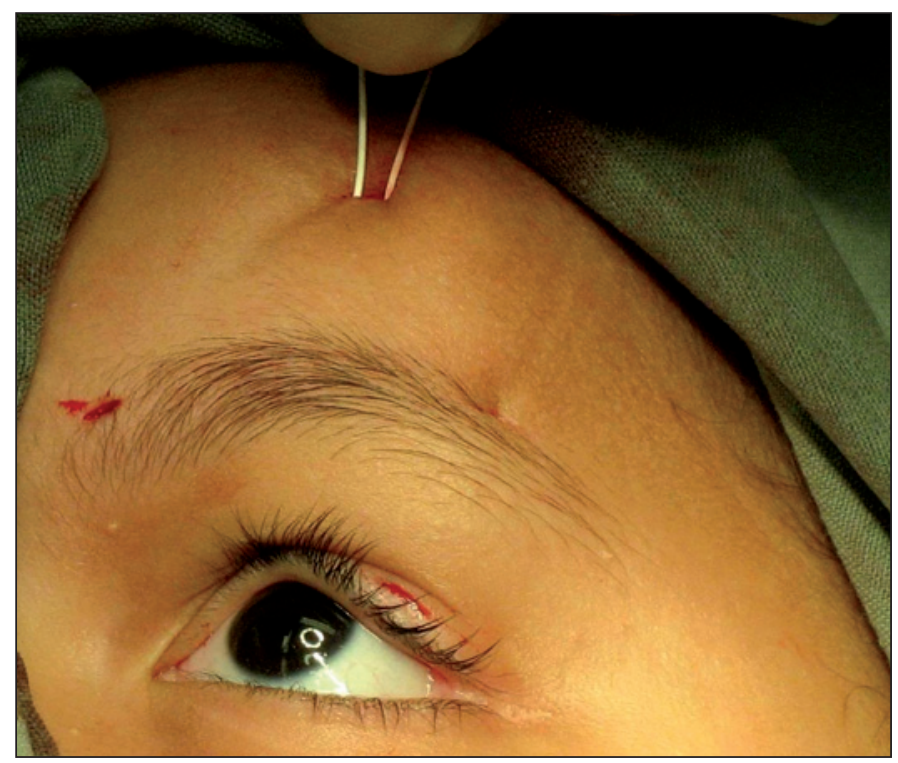

Figura 2 - Posição final com fio emergindo da incisão frontal superior
Fáscia lata autóloga já foi muito utilizada em procedimentos de suspensão ${ }^{(23-24)}$, e ainda é considerada como padrão ouro para o tratamento deste tipo de ptose, apesar de ter suas desvantagens. Dentre elas, a necessidade de um segundo sítio cirúrgico, em que muitas vezes o cirurgião não está familiarizado com a região e não isenta de riscos. Para crianças com menos de 4 anos, a fáscia lata é ainda irregular e fina para ser usada. Outra opção de fáscia é a temporal ${ }^{(25)}$, mas com praticamente as mesmas restrições da fáscia lata. Porém é preferível a utilização de material autólogo em casos de recidiva em crianças, e também nos casos em que os tecidos estão muito alterados como em algumas ptoses traumáticas e cavidades retraídas.

A fáscia lata heteróloga é mais problemática por apresentar um maior número de infecções e granulomas, além das recorrências e a possibilidade de conter vírus de tipo lento (Creutzfeld-Jacob) $^{(26)}$.

Outra possibilidade é o uso de materiais sintéticos como silicone, poliéster, nylon, supramida ${ }^{(17-19)}$, mas estes não são biointegráveis, sendo que o poliéster apresenta melhor resultado $^{(26)}$

O primeiro material que cumpre esta condição é o PTFEe, que tem demonstrado ter uma grande biocompatibilidade a longo prazo; não é antigênico e é inerte tanto biológica como quimicamente $^{(27-28)}$. O PTFEe apresenta porosidade de $50 \%$ do seu volume de ar, com bons resultados e uma colonização celular precoce. O estudo histológico do PTFEe mostra um tempo de colonização suficiente, que permite observar a presença de um novo estroma funcional composto por fibroblastos, células endoteliais e neovasos que se introduzem nos poros sem que se observe reação inflamatória, granuloma, reação a corpo estranho, necrose, ou lise ${ }^{(29)}$.

Os bons resultados ${ }^{(19)}$ com PTFEe indicam que se pode substituir a fáscia lata autóloga, evitando as complicações relacionadas à sua obtenção.
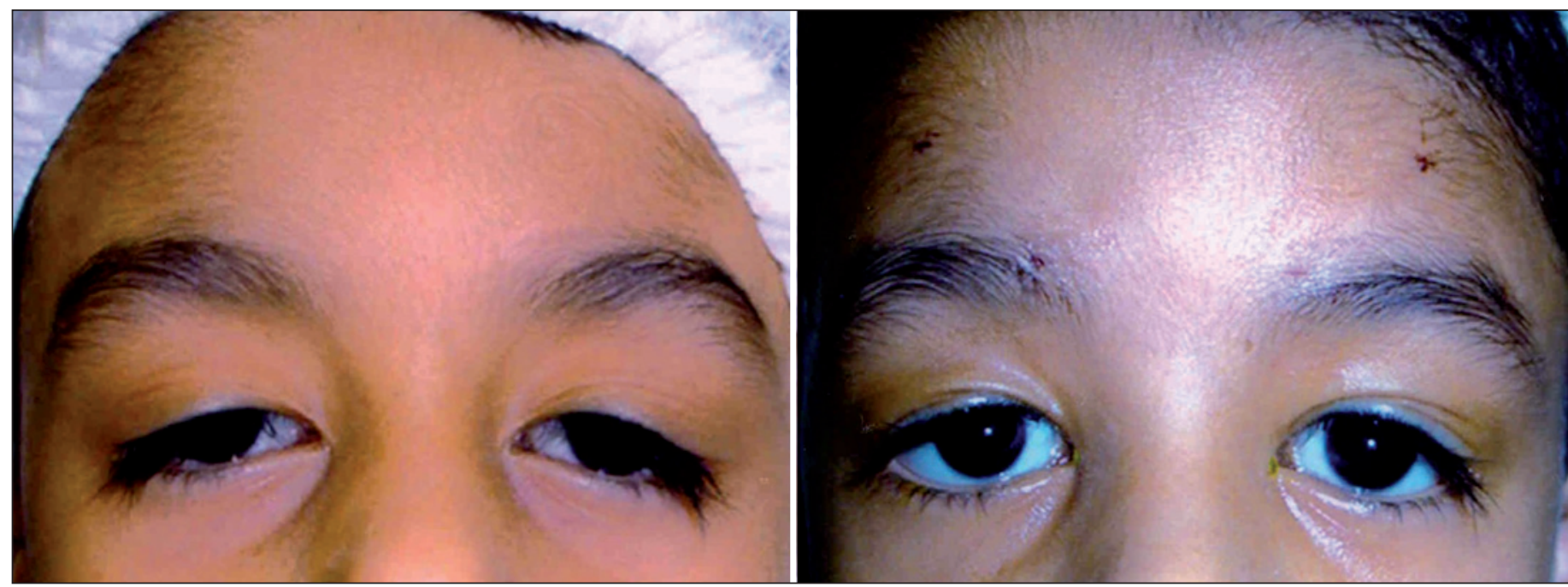

Figura 3 - Pré e pós-operatório da técnica de suspensão frontal bilateral com fio de Gore-Tex ${ }^{\circledR}$ Modelo CV3, 6.0 (W.L. Gore \& Associates Inc, Flagstaff, AZ, EUA) 
Como é um material estranho deve ser colocado corretamente entre os tecidos pálpebro-faciais e manipulado respeitando-se as regras de assepsia. Como o PTFEe é altamente poroso e pode sustentar bactérias contaminantes com formação de abscessos, recomenda-se suturar todas as incisões da pele ${ }^{(26)}$.

Em uma série de 37 casos de suspensão frontal usando PTFEe, acompanhadas por uma média de 3 anos, Steinkogler et al. ${ }^{(28)}$ reportaram um caso de recorrência da ptose necessitando nova cirurgia e um caso onde foi necessária a retirada do fio.

Apesar de o presente estudo envolver casos de ptoses graves, os resultados funcionais foram bons ou ótimos em mais de $85 \%$ dos casos. Apenas dois casos tiveram o fio removido, um devido a uma celulite facial na região frontal, na primeira semana de pós-operatório, por não seguir as recomendações de higiene e repouso, sendo necessária a sua retirada imediata, sem recolocação. Encontramos a formação de granuloma na pele da região frontal em $13,4 \%$ dos pacientes na primeira semana pós-operatória, com boa resposta ao tratamento conservador sendo que apenas um paciente ainda o apresentava após três meses, sendo necessária sua retirada, pela recorrência dos granulomas, com posterior recolocação. Embora reportado com pouca frequência, as complicações associadas com PTFEe em cirurgias de ptose são rejeição, formação de granuloma e recorrência da ptose ${ }^{(28)}$.

Em contraste com o resultado satisfatório encontrado neste estudo com o uso do PTFEe, os estudos de Wasserman et al. mostraram uma alta porcentagem $(45,5 \%)$ de infecção e/ou formação de granulomas no grupo que usou o PTFEe, fato atribuído à alta porosidade do material, que permite o sequestro de micro-organismos ${ }^{(14)}$.

O PTFEe apresenta uma dimensão que pode ser posicionada através de uma pequena incisão, o que reduz a extensão da cicatriz palpebral. A eleição do material mais adequado também dependerá dos resultados a longo prazo, sobretudo nas crianças.

\section{CONCLUSÃO}

O PTFEe - Modelo CV3, 6.0 (Gore-Tex ${ }^{\circledR}$; W.L. Gore \& Associates Inc, Flagstaff, AZ, EUA), monofilamento, na cirurgia de suspensão ao frontal pode ser uma alternativa para tratamento de ptose palpebral grave com função má ou nula do músculo levantador da pálpebra superior, sendo um material adequado com bons resultados funcionais $(86,9 \%)$, baixos índices de complicação $(4,35 \%)$ e insatisfação $(13,4 \%)$.

\section{ABSTRACT}

Purpose: To evaluate the functional results and complications of the use of expanded polytetrafluoroethylene in frontalis suspension surgery for the treatment of blepharoptosis. Methods: Frontalis suspension procedure with polytetrafluoroethylene was performed between 2003 and 2007 on 23 patients (36 eyes) with blepharoptosis. An average follow-up time was 15.8 months (range, 3 to 36). Surgical technique used was described by Fox. Results: The causes of blepharoptosis found were: congenital in 20 patients $(86.95 \%)$, blepharophimosis in $2(8.69 \%)$ and traumatic in 1 $(4.35 \%)$. At the first week of postoperatory $6(26.08 \%)$ patients related palpebral asymmetry, 4 (17.39\%) noticed local edema, $3(13.04 \%)$ presented granulomas and $1(4.35 \%)$ presented facial cellulitis on the ipsilateral frontal region. After 3 months of follow-up $3(13.04 \%)$ patients related palpebral asymmetry and $1(4.35 \%)$ persisted the granuloma. Conclusion: Polytetrafluoroethylene - Model CV3, 6.0 (Gore-tex $^{\circledR}$, W.L. Gore \& Associates Inc, Flagstaff, AZ, EUA) is an adequate material with good functional results $(86.9 \%)$, relatively few complications $(4.35 \%)$ and insatisfaction $(13.4 \%)$ that could be an alternative for fascia lata in the surgery of frontalis suspension for the treatment of blepharoptosis.

Keywords: Blepharoplasty/methods; Blepharoptosis/surgery; Polytetrafluoroethylene/utilization; Polytetrafluoroethylene/adverse effects

\section{REFERÊNCIAS}

1. Nesi FA, Lisman RD, Levine MR, editors. Smith's ophthalmic plastic and reconstructive surgery. St. Louis: Mosby; 1998.

2. Soares EJC, Figueiredo ARP, Souza GL, Almeida HC, Oliveira LRM, Magalhães MM, Portellinha WM. Blefaroptose. In: Soares EJC, Moura EM, Gonçalves JOR, editores. Cirurgia plástica ocular. São Paulo: Rocca; 1997. p.77-152.

3. Lucci LMD, Portellinha W, Sant'Anna AEBPP. Ptose palpebral: estudo de 390 casos. Arq Bras Oftalmol. 1997;60(5):455-7.

4. Merriam WW, Ellis FA, Helveston EM. Congenital blepharoptosis, anisometropia, and amblyopia. Am J Ophthalmol. 1980;89(3):401-7.

5. Hornblass A, Kass LG, Ziffer AJ. Amblyopia in congenital ptosis. Ophthalmic Surg. 1995;26(4):334-7.

6. Stärk N, Zubcov AA, Kast E, Gutermuth D. [Amblyopia, refractive errors and strabismus in congenital ptosis]. Ophthalmologe. 1996;93(4):345-50. German.

7. Fiergang DL, Wright KW, Foster JA. Unilateral or asymmetric congenital ptosis, head posturing, and amblyopia. J Pediatr Ophthalmol Strabismus. 1999;36(2):74-7.

8. Ugurbas $\mathrm{SH}$, Zilelioglu G. Corneal topography in patients with congenital ptosis. Eye. 1999;13(Pt 4): 550-4.

9. McCulloch DL, Wright KW. Unilateral congenital ptosis: compensatory head posturing and amblyopia. Ophthal Plast Reconstr Surg. 1993;9(3):196-200.

10. Finsterer J. Ptosis: causes, presentation, and management. Aesthetic Plast Surg. 2003;27(3):193-204.

11. Sant'Anna AEBPP, Genta CB, Portelinha W. Uso do PTFE (Gore-Tex) para tratamento da blefaroptose severa. Arq Bras Oftalmol. 1994;57(2):84-6.

12. Crawford JS. Repair of ptosis using frontalis muscle and fascia lata: a 20-year review. Ophthalmic Surg. 1977;8(3):31-40.

13. Wagner RS, Mauriello JA Jr, Nelson LB, Calhoun JH, Flanagan JC, Harley RD. Treatment of congenital ptosis with frontalis suspension: a comparison of suspensory materials. Ophthalmology. 1984;91(3):245-8.

14. Wasserman BN, Sprunger DT, Helveston EM. Comparison of materials used in frontalis suspension. Arch Ophthlamol. 2001;119(5):687-91.

15. Fox SA. Ophthalmic plastic surgery. $3^{\text {rd }}$ ed. New York: Grune \& Stratton; 1963.

16. Broughton WL, Matthews JG $2^{\text {nd }}$, Harris DJ Jr. Congenital ptosis. Results of treatment using lyophilized fascia lata for frontalis suspensions. Ophthalmology. 1982;89(11):1261-6.

17. Liu D. Blepharoptosis correction with frontalis suspension using a supramid sling: duration of effect. Am J Ophthalmol. 1999;128(6):772-3. 
18. Bernardini FP, de Conciliis C, Devoto MH. Frontalis suspension sling using a silicone rod in patients affected by myogenic blepharoptosis. Orbit. 2002; 21(3):195-8.

19. Bajaj MS, Sastry SS, Ghose S, Betharia SM, Pushker N. Evaluation of polytetrafluoroethylene suture for frontalis suspension as compared to polybutylate-coated braided polyester. Clin Experiment Ophthalmol. 2004;32(4): 415-9.

20. Adenis JP, Lebraud P, Mathon M. [Use of polytetrafluoroethylene (Goretex) in the palpebrofrontalis muscle suspension in ptosis]. J Fr Ophtalmol. 1987; 10(10):607-9. French.

21. Ruban JM, Mallem M, Tabone E, Donne C. [A new biomaterial in surgery of ptosis with frontalis suspension: wide pore PTFE]. J Fr Ophtalmol. 1995; 18(3):207-19. French.

22. Leibovitch I, Leibovitch L, Dray JP. Long-term results of frontalis suspension using autogenous fascia lata for congenital ptosis in children under 3 years of age. Am J Ophthalmol. 2003;136(5):866-71.

23. Crawford JS. Nature of fascia lata and its fate after implantation. Am J Ophthalmol. 1969;67(6):900-7
24. Crawford JS. Congenital blepharoptosis. In: Smith BC, Della Rocca RC, Nesi FA, Lusman RD, editors. Ophthalmic plastic and reconstructive surgery. St. Louis, MO: Mosby; 1987. p.643-5.

25. Nunes TP, Sardinha M, Cardoso L, Buzalaf F, Matayoshi S. Uso da fáscia temporal na suspensão frontal: descrição da técnica cirúrgica - Relato de caso. Arq Bras Oftalmol. 2004;67(2):323-7.

26. Mencía-Gutiérrez E, Clariana-Martín A, Gutiérrez-Díaz E, Monsalve-Córdova J, Izquierdo-Rodrigues C. Resultados y complicaciones del politetrafluoroetileno expandido en la cirugía de ptosis palpebral por suspensión al frontal. Estudio de 59 casos. Arch Soc Esp Oftalmol. 2005;80(8):443-8.

27. Zweep HP, Spauwen PH. Evaluation of expanded polytetrafluoroetylene (ePTFE) and autogenous fascia lata in frontalis suspension. A comparative clinical study. Acta Chir Plast. 1992;34(3):129-37.

28. Steinkogler FJ, Kuchar A, Huber E, Arocker-Mettinger E. Gore-Tex soft-tissue patch frontalis suspension technique in congenital ptosis and blepharophimosisptosis syndrome. Plast Reconstr Surg. 1993;92(6):1057-60.

29. Jeong S, Ma YR, Park YG. Histopathological study of frontalis suspension materials. Jpn J Ophthalmol. 2000;44(2):171-4. 